\title{
Les Cellules adipeuses
}

\author{
par \\ N. A. Ter-Ossipian
}

Chaire d'Histologie de l'Institut Vétérinaire Zootechnique d'Erivan, USSR, Titulaire de la Chaire: N. A. Ter-Ossipian, Directeur de l'Institut: Prof. B. G. Massino

Reçu le 14 az'ril 1937 *

\section{De la relation entre les cellules adipeuses et les éléments de la substance intercellulaire}

Chez les brebis du Caucase il se forme sous la peau, à la partie supérieure de la queue des dépôts de graisse sous forme d'excroissance. Un sillon longitudinal divise cette excroissance adipeuse en deux moitiés symétriques, entre lesquelles se disposent les vertèbres caudales. De chaque côté des vertèbres l'épaisseur de l'excroissance adipeuse est parcourue par des tractus fibreux riches en fibres élastiques qui soutiennent tout le poids de la graisse déposée. Les vertèbres caudales supérieures sont découvertes du côté dorsal. Par une section longitudinale du côté dorsal de l'excroissance adipeuse on peut pénétrer dans le canal rachidien ouvert où passe la gaîne de la dure-mère. Cette dernière est entourée par un tissu adipeux très lâche qui remplit le canal rachidien. Ce tissu, dont il est facile de préparer des lamelles minces, m'a servi dans mes recherches.

Ces lamelles avaient été fixées à l'aide de la formaline, du formol de Zenker et du sublimé alcoolisé. Elles furent ensuite colorées à la picrofuchsine, d'après Mallory, Unna, Belchovsky, par l'hématoxyline d'après Heidenhain etc. A part ces lamelles, nous avions fixé également des fragments prélevés sur le même tissu adipeux, plongés dans la paraffine ou la celloïdine et colorés d'après les mêmes méthodes.

Les recherches avaient également porté sur le tissu adipeux des embryons de brebis et de chats.

Par la suite on avait entrepris l'étude du tissu adipeux en général, chez le gros et petit bétail à cornes, chez le porc, le chat, l'homme. On a également étudié le tissu adipeux sous-cutané, le tissu intra-musculaire, périrénal ainsi que des autres régions de l'organisme.

Le fait que les épreuves corrigeés par l'auteur n'ont pas été reçues par la revue bien que l'envoi en ait été réitéré deux fois, est la cause du long délai qu'a subi la publication de cet article.

Le directeur de la revue. 
En étudiant la structure microscopique des tractus fibreux de l'excroissance adipeuse, mon attention a été attirée par le fait que de chaque cellule adipeuse incorporée dans ces tractus partait un assez gros faisceau de fibres collagènes ${ }^{1)}$ (v. fig. 1).

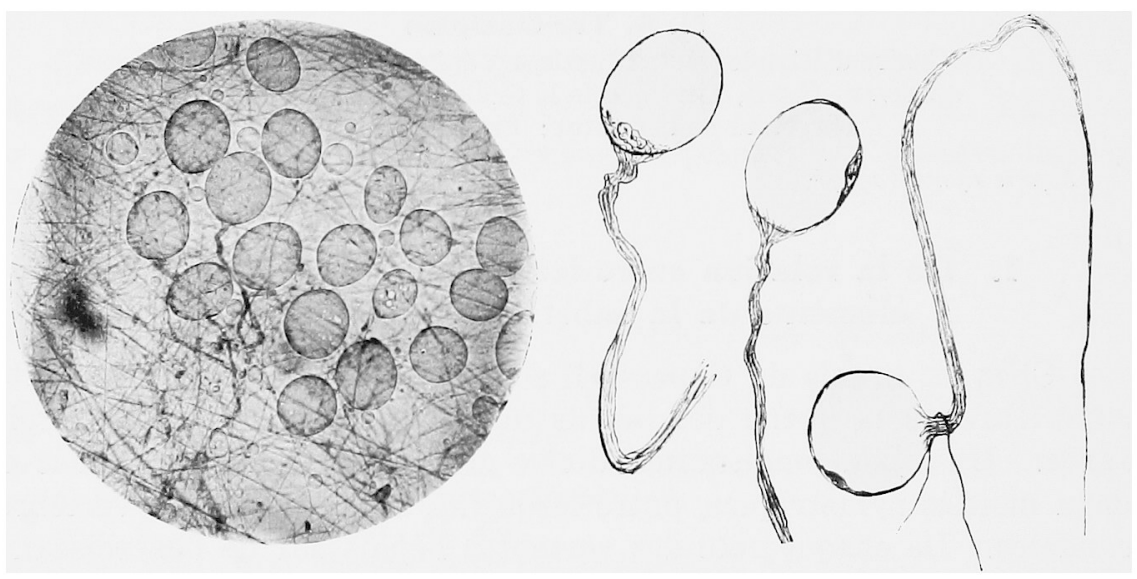

Fig. 1. Cellules adipeuses en liaison génétique avec les faisceaux de fibres collagènes. Coloré d'après Mallory. Les dessins ont été exécutés au moyen d'un appareil à dessin monté au niveau de la table du microscope.

Afin de se convaincre de l'existence réelle de cette relation qui fait supposer une relation génétique, une étude détaillée de toute l'excroissance adipeuse a été entreprise, étude qui a absorbé bien des efforts infructueux, vu l'impossibilité d’obtenir des préparations avec des cellules semblables. Ce n'est que bien des mois après qu 'il nous a été donné de les déceler lors d'une étude sur le tissu adipeux entourant la dure-mère.

Le tissu adipeux remplissant le canal dans la région des vertèbres caudales supérieures, grâce à la disposition lâche des cellules adipeuses résultant de l'abondance des éléments de mésenchyme et de tissu conjonctif, s'est montré particulièrement approprié pour les recherches, et c'est sur ce tissu que se sont portées nos observations principales.

1) Il faut remarquer que dans la littérature consacrée au tissu adipeux à part l'hypothèse de Plenk (selon laquelle la formation de la substance fondamentale doit être non seulement particulière aux cellules du tissu conjonctif dans le sens strict du mot, mais aussi aux autres éléments d'origine mésodermique, à savoir aux cellules adipeuses et endothéliales, aux fibres musculaires lisses et striées) on ne trouve aucune indication à ce sujet, si l'on ne compte pas le mémoire de Marchand qui avait étudié l'atrophie du tissu adipeux transplanté; dans son article (tableau 2, fig. 4) on voit des cellules déjà dépourvues de graisse, en relation topographique avec les faisceaux. Marchand trouve qu'en fait les cellules ne sont pas séparées des faisceaux. 
Sur les préparations faites avec ce tissu adipeux on voit clairement que chaque cellule adipeuse sert de point de départ à un faisceau de fibrilles formant un petit cône. Des fibrilles isolées partant de la surface de la cellule adipeuse se dirigent d'un seul côté et forment en convergeant un faisceau de fibres collagènes. Parfois, on réussit à suivre pendant longtemps le trajet d'un tel faisceau isolé. Dans la plupart des cas les faisceaux isolés partant des cellules avoisinantes se rapprochent en formant un faisceau commun (v. fig. 2). Le point de départ du faisceau ne dépend pas de la disposition du noyau, celui-ci se disposant au commencement ou à distance du faisceau. Etant dorné qu'avec la méthode des préparations en lamelles les relations morphologiques propres au tissu vivant peuvent être altérées, afin d'exclure tout doute possible nous avont étudié des coupes de fragments prélevés sur ce même tissu adipeux, et immergés dans la celloïdine ou la paraffine, ou bien des coupes préparées au moyen du microtome à congélation. Ces dernières conservent mieux la relation entre les cellules et le tissu conjonctif environnant. Etant donné qu'à l'immersion la préparation se contracte, par suite de l'extraction de la graisse, la membrane des cellules adipeuses se rétracte et les cellules ainsi rapprochées compriment les faisceaux fibrillaires. Cependant, même sur de telles coupes on peut voir presque dans chaque champ visuel une ou deux cellules, incluses par chance dans la coupe, et se trouvant en relation topographique avec le faisceau de fibres collagènes.

Après confirmation de cette observation il nous a paru évident que les cellules adipeuses du dit tissu n'étaient pas des unités libres, réunies en lobules, mais que chaque cellule envoyant son faisceau vers quelque faisceau commun était par cela-même liée aux autres cellules.

En étudiant la structure du tissu adipeux chez les autres animaux nous n'avions pas réussi au début à trouver des cellules avec des faisceaux de fibres partant de ces dernières, car dans les lobules les cellules sont disposées de manière très serrée. Par la suite, cependant, en imprimant de légers coups à la lamelle de la préparation on déplaçait les cellules adipeuses constituant le lobule ${ }^{1)}$ et de cette façon on parvenait à déceler le faisceau de sortie des cellules ainsi déplacées, qui était beaucoup moins développé que chez les cellules décrites plus haut (v. fig. 3).

Mêmes résultats avec le tissu adipeux brun des rongeurs.

Des observations analogues ont été faites sur des cellules adipeuses isolées, toujours situées près de quelque faisceau. Par petits

1) Dans ces cas la préparation fraîche était colorée par le tripanblau et examinée dans la glycérine. 
chocs imprimés à la lamelle on réussit à déplacer une telle cellule et à déceler son faisceau (v. fig. 4) .

$\mathrm{Si}$, par suite de ces chocs, la graisse s'écoule partiellement de la cellule, ce qui arrive très facilement avec la graisse humaine, à cause de la diminution de la turgescence, la membrane de la cellule adipeuse sous forme de sac affaissé reste reliée à sa tige fibreuse et s'en détache avec difficulté.

D'après Wassermann la séparation des régions nucléo-protoplasmiques en cellules adipeuses isolées n'a pas lieu, et seul le réticulum de la graisse brune donne des cellules adipeuses isolées de lipoblaste. Plenk trouve peu motivé le point de vue d'après lequel la formation des lipoblastes serait propre à la graisse brune seulement et fait remarquer que dans le tissu adipeux ordinaire on peut voir nettement des membranes isolées sur beaucoup de cellules, dont la graisse a été extraite, et que sur le vieux matériel fixé par le liquide de Müller les cellules du tissu adipeux peuvent être facilement isolées.

L'hypothèse que les cellules adipeuses sont des unités délimitées par la membrane et reliées seulement par leurs faisceaux, est con-

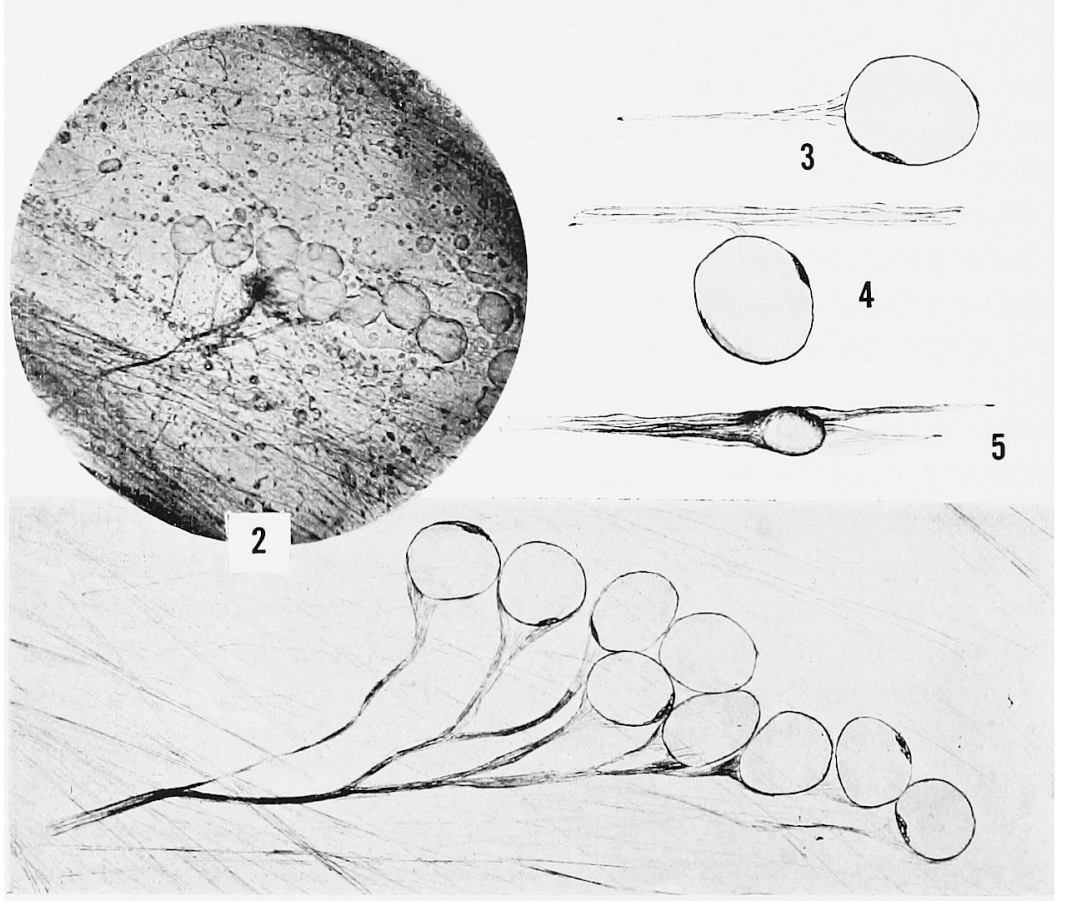

Fig. 2-5. 2. Groupe de cellules adipeuses envoyant ses fibres à un faisceau commun. Coloré d'après la méthode d'Unna. 3. Cellule adipeuse séparée du lobule du tissu cellulaire sous cutané de l'homme. Coloré par le bleu tripanblau. 4. Cellule adipeuse isolée déplacée. Coloré au bleu tripanblau. 
firmée par les observations exposées ci-dessus ainsi que par le fait que sous l'influence de l'action mécanique, des chocs imprimés à la lamelle de la préparation, les cellules s'écartent les unes des autres et se laissent difficilement détacher de leur propre faisceau. Si la cellule adipeuse avait été de toutes parts en relation syncitielle, alors nous n'aurions pas observé constamment son isolement de tous les côtés, sauf un.

Un fait plus important est la relation entre le faisceau qui se dirige vers la membrane et entre cette dernière et la cellule adipeuse.

Dans la membrane des cellules adipeuses se trouve ordinairement un fin réseau fibrillaire que Plenk considère à juste titre comme pratiquement inséparable de la membrane.

Plenk fait remarquer que les fins filaments de la membrane dans le tissu adipeux embryonnaire noircissent sous l'action de l'argent tandis que dans les cellules adipeuses adultes ces filaments gardaient leur couleur brun fauve.

Les travaux d'un grand nombre d'auteurs ont démontré que les fibres argyrophiles et précollagènes se transformaient directement en fibres collagènes ${ }^{1)}$. Laquesse, Maximoff, Alféeff et les autres montrent qu'à leur apparition les fibres collagènes passent constamment par la phase argyrophile tandis que Petersen, Plenk et Iassvoin estiment que ce stade n'êst pas obligatoire et admettent que dans la plupart des cas les fibres collagènes à leur apparition échappent à la phase argyrophile. ${ }^{2)}$

Quant à l'importance de la membrane de la cellule adipeuse, Bell en 1909 a indiqué que la membrane prend son origine dans le plasma périphérique cellulaire et que dans l'atrophie des cellules adipeuses la membrane se conserve. En 1920 Marchand, qui avait étudié le tissu adipeux transplanté, a indiqué que la membrane des cellules adipeuses prend la coloration de la même manière que le tissu conjonctif à l'intérieur des lobules adipeux. Cet auteur compare la membrane des cellules adipeuses au sarcolemme des fibres musculaires et à la capsule des cellules cartilagineuses. Policard en 1922 identifie la membrane à la substance collagène. Grinfeld (1922) et Wassermann (1926) acceptant la structure syncitielle du tissu adipeux esti-

1) Bauer (1934) nie l'opinion générale admise selon laquelle les fibres argyrophiles ne seraient qu'une forme juvénile des fibres collagènes.

2) Iassvoïn (1934) distingue deux périodes dans le processus de la formation des fibres collagènes. Pendant la première période le synectoplasme du mésenchyme renferme des fibres argyrophiles, qui se transforment en fibres collagènes. A la deuxième période il n'y a pas de fibres argyrophiles et l'ectoplasme amorphe se transforme directement en substance basale, au lieu de transition les fibres viennent de l'ectoplasme directement sous forme de fibres collagènes. 
ment que les cellules adipeúses n'ont pas de membrane spéciale. Plenk en 1927 trouve que la membrane doit être identifiée avec la substance collagène du tissu conjonctif, étant donné qu'elle contient des fibres. Selon l'avis de Plenk, la substance collagène du tissu conjonctif est le produit de la sécrétion des cellules, où se forment les fibres.

Nous avons eu constamment l'occasion de constater que certaines cellules adipeuses avaient une membrane particulièrement épaisse, donnant d'ailleurs l'impression d'être lamelleuse; plus cette membrane devient épaisse, plus les dimensions de la cellule elle-même diminuent par suite de la réduction de la quantité de graisse accumulée (v. fig. 5).

Nous avons observé enfin des formations qui ne contenaient point de graisse du tout et qui se présentaient sous la forme d'un petit ovale épaissi au bout du faisceau collagène. A la picrofuchsine cette formation prend une couleur rouge foncé, et parfois l'on voit nettement sa structure fibrillaire.

Nous estimons que du fait que la membrane des cellules adipeuses prend part à la fibrogénèse elle doit être reconnue comme ectoplasme; mais au fur et à mesure que la cellule adipeuse devient plus âgée, la membrane se transforme en métaplasme, et ce processus s'étend graduellement à toute la cellule.

Une série d'auteurs ont signalé aussi que les cellules produisant la substance intercellulaire diminuaient assez souvent de volume; parfois même leur protoplasme peut s'user complètement; alors, on voit ces cellules s'atrophier et disparaître.

Laquesse a décrit la transformation progressive des parties périphériques des cellules du tissu conjonctif en substance précollagène. Lorsque cette transformation embrasse toute la cellule, cette dernière se détruit avec des phénomènes de caryolyse. Le même fait est indiqué par Schafer pour les cellules cartilagineuses, et par Iassvoïn - pour les cellules osseuses.

Comme on le sait, il existe actuellement plusieurs opinions concernant la formation de la substance intercellulaire. Laquesse, Studnicka, Zavarzin, Iassvoïn et les autres soutiennent la théorie de la transformation de l'ectoplasme des cellules. Petersen, Plenk et les autres défendent la théorie de la sécrétion, c'est-à-dire ils estiment que les cellules sécrètent une substance amorphe, au sein de laquelle apparaît la structure fibrillaire. Enfin, Nageotte et certains autres auteurs se prononcent pour l'origine extracellulaire de la substance intercellulaire.

Comme il a été déjà indiqué plus haut, en étudiant nos préparations nous avons eu plus d'une fois l'occasion d'observer l'accroisse- 
ment progressif de la membrane de la cellule adipeuse à l'intérieur; la diminution de la goutte graisseuse et, enfin, la résorption complète du protoplasme et la disparition du noyau. L'extrémité du faisceau collagène, où se trouvait auparavant la cellule, prend la forme d'un petit ovale compacte, coloré par la picrofuchsine en rouge foncé. ${ }^{11}$ S'il y avait ici des phénomènes de sécrétion, alors dans les dernières phases de transformation, quand la vitalité du protoplasme et du noyau s'éteint, la sécrétion serait troublée et l'on n'observerait pas une transformation complète en métaplasme, car selon toute probakilité ce ne sont que les cellules saines et en pleine vitalité qui soient aptes à la sécrétion.

L'épaississement de la couche enveloppant la goutte graisseuse, la réduction graduelle de la quantité de graisse accumulée jusqu'à disparition complète, et surtout la disparition du protoplasme et sa transformation en métaplasme, - tous ces faits parlent en faveur de la transformation et non de la sécrétion.

En tenant compte de ce que les cellules adipeuses sont liées à la substance intermédiaire comme l'on peut s'en convaincre sur nos préparations, l'opinion touchant la séparation du métaplasme d'avec les cellules tombe d'elle-même.

\section{Transformation des substances du noyau de la cellule adipeuse en cytoplasme}

Comme on le sait, la formation de la graisse dans les cellules est un processus biochimique complexe. Les recherches de Flemming, de Metzner et autres ont établi que l'apparition des gouttelettes de graisse dans le protoplasme est précédée par la formation de grains menus autour desquels la graisse se dépose. Par la suite, l'étude de la nature chimique de ces grains a permis de les considérer comme formations albuminoïdes. ${ }^{2}$

Flemming a constaté aussi des trous à-jour dans les noyaux des cellules adipeuses. Râble attribua ce phénomène à la présence d'une goutte de graisse dans le noyau qui, traitée par les alcools, se dissout et simule un orifice.

En hiver, on trouve un peu plus rarement sur notre matériel des noyaux ayant un orifice. Leur nombre augmente brusquement à la fin du printemps quand les brebis commencent à accumuler la graisse. On voit sur les préparations colorées au soudan III que l'orifice est rempli par une goutte de graisse; cette goutte apparait au centre du

1) La picrofuchsine colore les fibres précollagènes en rose et les fibres collagènes - en rouge.

2) A la suite de la réaction à la xanthoprotéine les grains jaunissent. 
nucléole et augmente progressivement tandis que le nucléole entoure la goutte en ceinture. Quand les dimensions de la goutte graisseuse augmentent fortement, le noyau prend la forme d'un anneau, et enfin, par suite de la rupture de la paroi du noyau, la goutte s'écoule dans le protoplasme. Alors le noyau et le nucléole reprennent leur forme primitive. En été, quand les brebis accumulent la graisse avec intensité dans leur excroissance, on trouve outre des noyaux à un seul orifice des noyaux à plusieurs orifices, dont le plus grand, appartenant au nucléole, a des contours plus nets (v. fig. 6).

Berg (1934), décrivant ses observations sur les noyaux des cellules hépatiques, établit également une relation entre la formation de la graisse et les nucléoles.

Dans nos préparations, parmi les cellules adipeuses on trouve également des cellules dont les noyaux sont complètement envahis par les menus grains ci-dessus mentionnés. Ces derniers sont colorés par l'hématoxyline d'après la méthode de Weigert et par l'argent d'après la méthode de Belchovsky; ils ont à peu près les mêmes dimensions. Parmi ces grains on peut discerner un ou deux grains plus grands correspondant aux nucléoles (v. fig. 7). Ces nucléoles contiennent une gouttelette de graisse ou en sont exempts.

Quand ces grains augmentent quelque peu ils sont rejetés dans le protoplasme où ils se trouvent disposés autour du noyau (v. fig. 8) ;
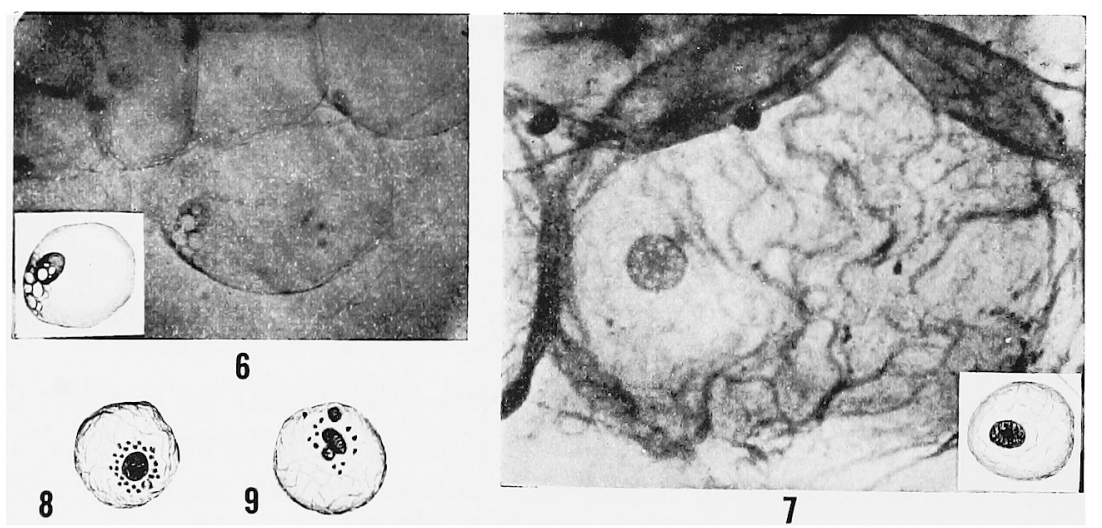

Fig. 6-9. 6. Cellule adipeuse à membrane épaiseie. Coloré d'après la méthode d'Unna. 7. Gouttes de graisse dans le noyau. Coloré à la picrofuchsine. 8. Noyau d'une cellule adipeuse renfermant des grains. Traité d'après la méthode de Belchovsky. 9. Grains rejetés dans le cytoplasme. Traité d'après la méthode de Belchovsky.

ensuite, ils commencent à s'écarter un peu, et l'on voit apparaître au centre de chaque grain une gouttelette de graisse, tandis que la substance composant le grain diffuse dans le protoplasme de la cellule adipeuse; c'est pourquoi cette dernière acquiert une teinte basophile et quelquefois se vacuolise. Chaque vacuole correspond à la goutte 
de graisse renfermée dans un grain. Ces cellules sont décrites sous le nom de cellules jeunes. En réalité, grâce à ce processus, la cellule est, pour ainsi dire, rajeunie. ${ }^{1)}$ Parfois, des nucléoles sont rejetés en même temps que les grains (v. fig. 9).

De cette façon, la graisse soit s'accumule dans le noyau avec la participation des nucléoles et des grains, soit les grains mûrs sont rejetés du noyau, dans le protoplasme, où il se forme à l'intérieur de chaque grain un goutte de graisse, ce qui donne au protoplasme de la cellule adipeuse un aspect vacuolisé.

Nous avons eu également l'occasion d'observer l'instant même de la rupture de la paroi du noyau lorsque la goutte de graisse accumulée dans le nucléole se déversait dans le protoplasme. Dans ce cas les bords de la déchirure de la paroi du noyau sont également resserrés par l'enveloppe.

\section{Multiplication des cellules adipeuses}

A l'heure actuelle, selon l'opinion généralement admise en ce qui concerne la multiplication des cellules adipeuses, celles-ci cesseraient de se multiplier dès que commence à s'accumuler la graisse. L'accroissement du tissu adipeux se produit aux dépens des dimensions des cellules adipeuses et grâce à la participation d'un nombre toujours plus grand de cellules dans le processus d'accumulation de la graisse. ${ }^{2)}$

En étudiant mon matériel de tissu adipeux j'ai pu me convaincre du fait de la multiplication des cellules adipeuses adultes par étranglement, ce qui a lieu de la manière suivante: le noyau de la cellule adipeuse correspondante augmente un peu, se colore plus intensivement et l'on voit s'accumuler des grains. Lorsque ces derniers parviennent dans le protoplasme, les nucléoles y sont rejetés également. Bientôt après à l'intérieur de chaque nucléole il se forme une goutte de graisse. A cause de la substance granuleuse le protoplasme devient nettement basophile. La goutte de graisse dans le nucléole augmente rapidement et est ceinturée d'un anneau nucléolaire, qui se

1) Une telle cellule peut commencer à se diviser.

2) En son temps, Flemming a décrit que dans l'atrophie séreuse des cellules adipeuses à la place de la goutte de graisse il se formait une accumulation d'un liquide muqueux tandis que le protoplasme avec le noyau se déplaçait vers le centre et parfois se divisait par division directe, par suite de quoi l'ancienne cavité de l'enveloppe se trouvait occupée par de petites cellules de forme angulaire. Flemming donna à cette forme d'atrophie le nom d'atrophie à multiplication. Par la suite, Maximoff expliqua ce phénomène par l'invasion de polyblastes dans les cellules adipeuses atrophiées. Nous avons également réussi à voir plus d'une fois des cellules adipeuses complètement envahies par les polyblastes. Parfois, les polyblastes se trouvaient en état de dégénérescence avec noyau faiblement coloré et protoplasme alvéolé. 
colore en noir intense par l'hématoxyline d'après Heidenhain. (v. fig. 10).

C'est probablement parce qu'elle est séparée par une cloison protoplasmique de la graisse de la cellule-mère que cette goutte graisseuse, s'accroissant de côté, ne se confond pas avec la graisse maternelle; par suite de quoi, la cellule adipeuse fait, à cet endroit, une saillie en forme de bourgeon, tandis que le noyau l'enserre à sa base par un large anneau foncé (fig. 11). Enfin, la partie saillante atteint les dimensions d'une cellule adipeuse ordinaire. Cette formation se compose alors de deux moitiés presque égales, partagées par l'anneau nucléolaire; de chaque côté de cet anneau on voit s'accumuler le protoplasme basophile parsemé de menues gouttelettes de graisse. Vers ce temps il ne reste le plus souvent qu'un gros noyau fortement coloré, qui se dispose toujours de telle sorte sur l'étranglement qu'une partie se trouve d'un côté, et l'autre-de l'autre (v. fig. 12). Ensuite, le noyau se divise amitotiquement en deux, et chaque moitié

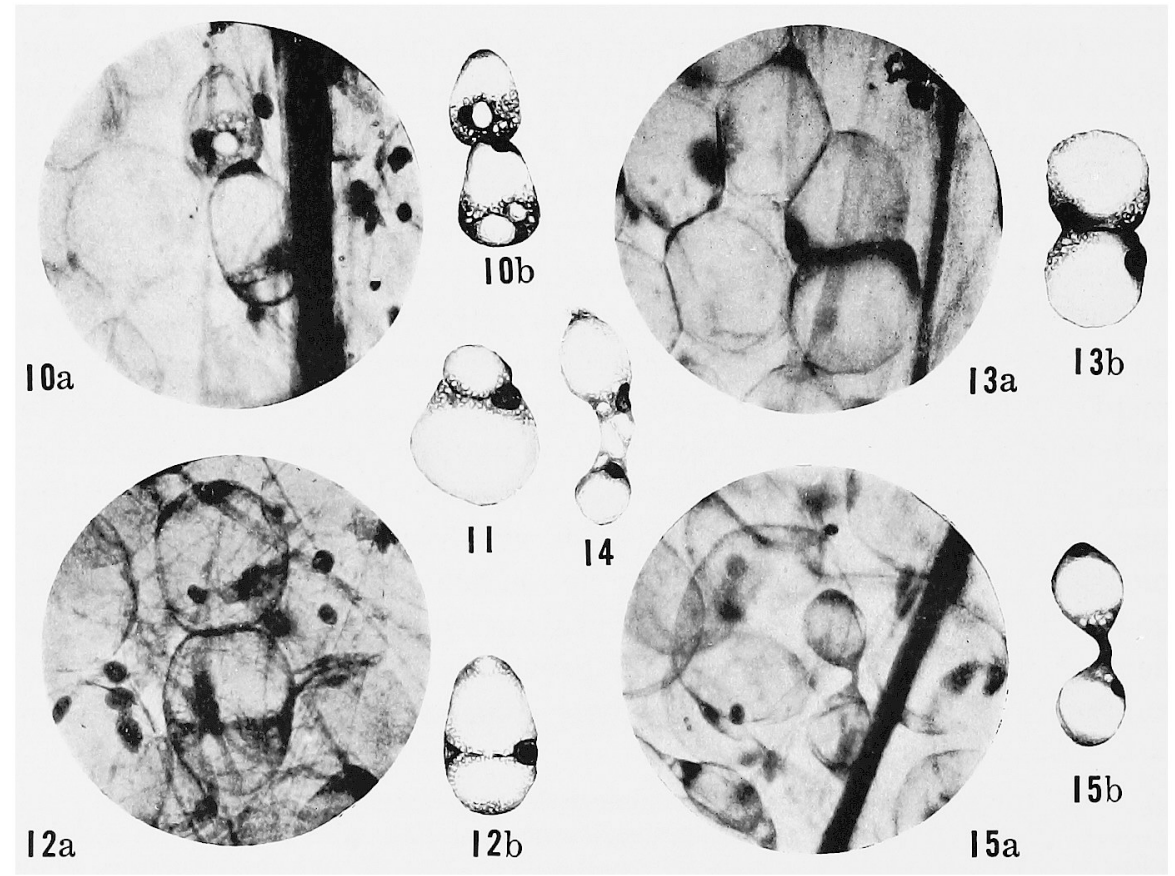

Fig. 10-15. 10. Nucléolus et grains dans le cytoplasme d'une cellule adipeuse. Traité d'après la méthode de Belchovsky. 11. Cellules adipeuses jeunes renfe mant une grande goutte de grais ce, entourée par l'anneau nucléolaire. Coloré à l'hématoxyline d'après la méthode de Heidenhain. $12 \& 13$. Phases successives de la croissance d'une goutte de graisse dans un nucléole. Coloré à l'hématoxyline d'après la méthode de Hei entain. 14. Cellules-filles adipeuses avec un noyau chacune. Coloré à l' hématoxyline d'après la méthode de Heidenhain. 15. Cellules adiceuses divisées, liées par des tractus protoplasmiques. Coloré par l'hématoxyline d'après la méthode de Heidenhain. 
de la double cellule reçoit son propre noyau (v. fig. 13). Quand sous l'influence de causes mécaniques les cellules - filles divergent un peu, elles se trouvent reliées au début par une tige protoplasmique (v. fig. 14, 15). Quand ce lien se trouve rompu, chaque cellule garde un pont protoplasmique basophile plus ou moins long (v. fig. 16). Par la suite ce pont protoplasmique se transforme en faisceau collagène et continue à s'accroître aux dépens de la cellule, ce qui est prouvé par l'existence de cellules adipeuses disposées aux deux extrémités d'une même faisceau collagène (v. fig. 17). Nous expliquons cette circonstance par le cas, où les cellules adipeuses tout en s'étant divisées n'ont pas rompu leur lien protoplasmique; celuici, transformé en faisceau collagène, continue à s'accroître reliant toujours entre elles deux cellules adipeuses.

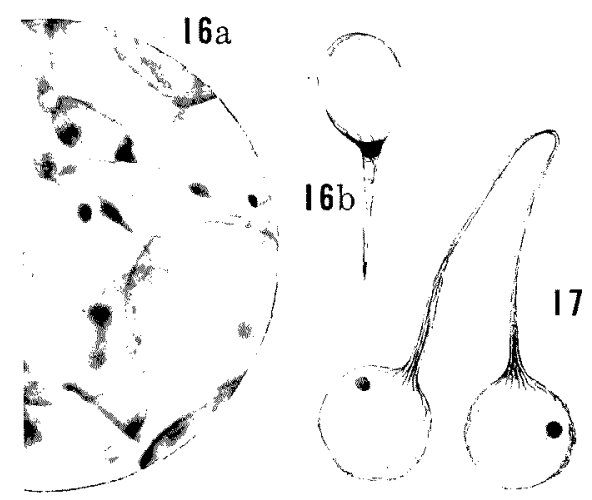

Fig. 16-17. 16. Protubérance protoplasmique d'une cellule adipeuse divisée. Coloré par l'hématoxyline d'après la méthode de Heidenhain. 17. Deux cellules adipeuses liées par un seul faisceau collagène. Traité par la méthode de Belchovsky.

Sans m'arrêter à la question de savoir si l'amitose est ou non un processus de dégénérescence, je noterai seulement qu'en ces derniers temps la division amitotique prend une assez grande expansion et ce phénomène est rattaché à un état fonctionnel particulier du protoplasme. D'après les observations de Mölendorff (1926) les amitoses se trouvent être le procédé ordinaire de division des noyaux dans les cultures croissantes des tissus, lorsqu'ils sont de nature symplastique. D'après Münzer (1925) le nombre des amitoses augmente avec une nourriture grasse et surtout albumineuse.

En résumant tout ce qui a été exposé plus haut, nous en venons à la conclusion qu'à côté de la participation de nouvelles cellules dans le processus d'accumulation graisseuse, il existe aussi une multiplication directe des cellules adipeuses déjà adultes. Les protubérances protoplasmiques des cellules divisées se transforment en faisceaux collagènes qui continuent de s'accroître aux dépens de la cellule, c'està-dire que la cellule adipeuse transformant continuellement une partie de son protoplasme croissant en substance intercellulaire reste liée à cette dernière.

Puisque cela est particulier aux cellules divisées, cela doit l'être également pour les cellules adipeuses qui proviennent de la différen- 
ciation des cellules restantes du mésenchyme; autrement dit, leur membrane, ayant reçu une structure fibrillaire, doit naturellement se continuer par un faisceau.

Il nous reste encore à élucider quels sont les facteurs qui déterminent telle ou telle direction du faisceau. De même, nous n'avons pas pu expliquer si la cellule se libérait du faisceau avant sa division, ou bien si elle passait à l'amitose tout en restant liée au faisceau.

\section{Literature}

1. Bauer, K. Zschr. d. mikr. anat. Forsch. Bd. 35, 1934.

2. Bell, E. Americ. Journ. of Anat. 1909.

3. Berg, W. Zschr. d. mikr. anat. Forseh. Bd. 35, 1934.

4. Flemming. Arch. f. Anat. u. Physiol. Anat. 1879.

5. Gryfellt, E, Communic. à la Soc. des Sciences Méd. et Biol. 1929.

6. Iassvoïn, G. Arch. f. mikr. Aanat. Bd. 102, 1924.

7. Zschr. f. mikr. anat. Forsch. Bd. 15, 1928.

8. - Arch. d'anat. micr. t, 30. No. 3, 1934.

9. Laquesse, E. Arch. d'anat. micr. t. 4, 1903.

10. Maximoff. Précis d'Histologie.

11. Marchand. Beitr. z. pathol. Anat. u. z. allg. Pathol. 66, 1920.

12. Mölendorff, W. Zschr. f. Zellf. u. mikr. Anat. 1926.

13. Nageotte, I. Compte-rendu des séances de la soc. de biol. t. 79, 1916.

14. - Ibid. t. 82, 1919 .

15. Ibid. t. 87,1922 .

16. Petersen, H. Hystologie und mikr. Anat. Bermann, 1924.

17. Plenk, H. Ergebnisse der Anatomie und Entwicklungsgeschichte, 1927.

18. Policard, A. Compte-rendu des séances de la soc. de biol. t. 74. II. 1922.

19. Studnicka, F. Anat. Anz. Bd. 22, 1906.

20. - Ebenda, Bd. 47, 1914.

21. Tello, F. Zschr. f. Anat. u. Entwick. 65, 1922.

22. Wassermann, N. Zschr. f. Zellf. u. mikr. Anat. Bd. 3, H. 2, 1926.

23. Zawarzin, A. Zschr. f. anat. Forsch. Bd. 6, 1926.

24. Cours d'histol. génér. 1932. 